\title{
SUSPENSÃO DE CIRURGIAS EM UM HOSPITAL UNIVERSITÁRIO
}

\author{
Suspension of surgery at a university hospital \\ Suspensión de la cirugía en un hospital universitario \\ Thialla Andrade Carvalho', Cinthia Barreto Sobral'2, Pabliane Matias Lordelo Marinho³, \\ Eliana Ofélia Ofélia Llapa-Rodriguez ${ }^{4}$, Maria Pontes de Aguiar Campos ${ }^{5}$
}

RESUMO: Objetivos: Analisar a incidência de suspensão de cirurgias, categorizar os cancelamentos cirúrgicos por especialidades médicas e identificar as suas principais causas. Método: Estudo quantitativo, descritivo, retrospectivo, realizado em um hospital de ensino do nordeste brasileiro. A população foi constituída por 1.600 cirurgias eletivas programadas no período de janeiro a setembro de 2013. A análise dos dados foi realizada através de estatística descritiva. Resultados: A taxa global de cancelamento de procedimento cirúrgico foi de 19,5\%. As maiores frequências de suspensão ocorreram nas cirurgias pediátricas, oncológicas e gerais. Foram identificadas 23 causas para o cancelamento de cirurgias na instituição, dentre elas destacaram-se o absenteísmo do paciente e as condições institucionais, representadas principalmente por problemas com recursos materiais, humanos e organização do serviço. Conclusão: A taxa de cancelamento de cirurgia remete à necessidade de reduzi-la; para tal, faz-se mister o monitoramento contínuo desse indicador e a implementação de estratégias para sua redução

Palavras-chave: Centro cirúrgico hospitalar. Cirurgia geral. Enfermagem perioperatória. Indicadores de qualidade em assistência à saúde.

ABSTRACT: Objectives: To analyze the incidence of surgery suspension, to categorize surgical cancellations into medical specialties, and to identify its main causes. Method: Quantitative, descriptive, and retrospective study carried out in a teaching hospital in the Northeast region of Brazil. The population was composed of 1,600 elective surgeries scheduled from January to September in 2013. Data analysis was performed through descriptive statistics. Results: The overall rate of surgical procedure cancellation was $19.5 \%$. The most frequent suspensions occurred in pediatric, oncology, and general surgeries. Twenty-three causes for surgery cancellation were found in the institution, amongst them patients' absence and institutional conditions represented mainly by problems with material, human, and organization service-related resources. Conclusion: The rate of surgery cancellation refers to the need of reducing it; for such, it is necessary to monitor this indicator continuously and to implement strategies for its reduction.

Keywords: Surgery department, hospital. General surgery. Perioperative nursing. Quality indicators, health care.

RESUMEN: Objetivos: Analizar la incidencia para suspensión de cirugías, categorizar las cancelaciones quirúrgicas por especialidades médicas e identificar sus principales causas. Método: Estudio cuantitativo, descriptivo, retrospectivo realizado en un hospital universitario del noreste del Brasil. La población constituida por 1.600 cirugías programadas de enero a septiembre de 2013. El análisis de datos se realizó utilizando estadística descriptiva. Resultados: La tasa global para cancelación de cirugías fue de 19,5\%. Los mayores porcentajes de suspensión fueron encontrados en las cirugias pediatricas, oncologícas y las generales. Se identificaron 23 causas para cancelación de cirugías en la institución, entre ellas se destacaron la ausencia del paciente y las condiciones institucionales, representadas principalmente por problemas con recursos materiales, humanos y organizacionales. Conclusión: Es necesario controlar e implementar estrategias para reducir la taxa de suspension encontrada.

Palabras clave: Servicio de cirugía en hospital. Cirugía general. Enfermería perioperatoria. Indicadores de calidad de la atención de salud.

\footnotetext{
'Mestranda em Enfermagem pela Universidade Federal de Sergipe (UFS); professora substituta do Departamento de Enfermagem da UFS - Aracaju (SE), Brasil. E-mail: thiallaba@hotmail.com Rua Maye Bell Taylor, 151 - Luzia - CEP: 49045-030 - Aracaju (SE), Brasil.

${ }^{2}$ Graduanda em Enfermagem pelo Departamento de Enfermagem da UFS - Aracaju (SE), Brasil.

${ }^{3}$ Mestre em Enfermagem pela UFS - Aracaju (SE), Brasil.

¿Doutora em Enfermagem Fundamental pela Universidade de São Paulo (USP); professora do Departamento de Enfermagem da UFS - Aracaju (SE), Brasil.

${ }^{5}$ Doutora em Enfermagem pela USP; professora do Departamento de Enfermagem da UFS - Aracaju (SE), Brasil.

Recebido: 16 jun. 2016 - Aprovado: 27 set. 2016

DOI: $10.5327 / Z 1414-4425201600040002$
} 


\section{INTRODUÇÃO}

A realização de um procedimento cirúrgico envolve ampla utilização de recursos humanos, materiais e tecnológicos, além de promover alterações no perfil psicológico e nos recursos financeiros dos pacientes e familiares ${ }^{1}$.

Os custos desses procedimentos correspondem a $40 \%$ do total das despesas de um hospital ${ }^{2}$, de modo que uma gestão inadequada do bloco operatório tem um impacto significativo nas unidades prestadoras de serviços de saúde, especialmente em instituições públicas onde os recursos são escassos ${ }^{3}$.

Assim, para subsidiar as tomadas de decisão dos gestores e para que seja prestada uma assistência de qualidade, com nível de excelência profissional, uso eficiente de recursos, mínimo de risco ao paciente/ cliente e alto grau de satisfação do usuário, faz-se necessário que o desempenho da unidade cirúrgica seja medido 4 .

Um dos indicadores utilizados para avaliar a eficiência de um serviço de cirurgia é a taxa de suspensão de procedimentos, que considera todos os motivos de interrupção, tanto os relacionados ao paciente quanto à instituição hospitalar 5 .

Na última década, o tema tem recebido grande atenção por parte dos pesquisadores da área da saúde no mundo ${ }^{6}$. Contudo, é necessária uma ampliação do olhar para compreender as perspectivas de todos os fatores envolvidos nesse processo e identificar suas causas, a fim de melhorar a qualidade do serviço prestado e aliviar o sofrimento do paciente e da família ${ }^{7}$.

$\mathrm{Na}$ literatura internacional encontramos taxas de suspensão de cirurgias divergentes, que variam de $0,37 \%$ (encontrada em hospital de Taiwan ${ }^{8}$ ) a $28 \%$ (encontrada em estudo nigeriano ${ }^{6}$ ).

No Brasil, estudo de revisão que avaliou as publicações no período de 1990 a 2010 identificou taxas de suspensão de cirurgia que variaram de 5,1 a $33,0 \%$, sendo que as principais causas foram referentes ao próprio cliente, seja por falta de condições clínicas, seja pelo não comparecimento para internação ${ }^{9}$. Pesquisa posterior, que avaliou os motivos de suspensão de cirurgia utilizando o método de análise da causa raiz, revelou como principais motivos: material inadequado (42,0\%); material com sujidade (29,0\%); falta de sala cirúrgica $(12,9 \%)$; falta de anestesista $(9,7 \%)$; e condições do paciente $(6,4 \%)^{10}$.

Diante das repercussões que o cancelamento cirúrgico traz para os usuários e instituições hospitalares e da importância desse indicador para o gerenciamento do bloco operatório, surge o seguinte questionamento: "Qual é a frequência e as principais causas de suspensão de cirurgias em um hospital universitário do nordeste do Brasil”?

\section{OBJETIVO}

Assim, o presente estudo teve como objetivos analisar a incidência de suspensão de cirurgias em um hospital universitário do nordeste brasileiro, categorizar os cancelamentos cirúrgicos de maior ocorrência por especialidades médicas e identificar as principais causas de cancelamentos de cirurgias em um hospital universitário.

\section{MÉTODO}

Estudo retrospectivo de natureza descritiva com abordagem quantitativa, realizado em um hospital de ensino de médio porte, que presta assistência médico-hospitalar de média complexidade, sendo referência no Sistema Único de Saúde (SUS).

A instituição possui 123 leitos, incluindo 5 de Unidade de Terapia Intensiva (UTI). O centro cirúrgico possui 4 salas cirúrgicas destinadas a procedimentos eletivos de segunda à sexta-feira, das 7 h00 às 19 h00, realizando uma média de 200 procedimentos cirúrgicos por mês. As especialidades cirúrgicas são: maxilo-facial; cabeça e pescoço; geral; aparelho digestivo; oncológica; pediátrica; plástica; mama; ginecológica; urológica; intestino; reto; e ânus.

A população foi constituída por todas as cirurgias de caráter eletivo, inclusive as ambulatoriais realizadas no centro cirúrgico, programadas no período de janeiro a setembro de 2013. A escolha por tal recorte temporal se deu em razão de, em janeiro daquele ano, ter sido institucionalmente implantado um instrumento impresso sistematizado para registro das informações relacionadas às suspensões de cirurgias.

Foram excluídos os prontuários cujos procedimentos cirúrgicos foram realizados no ambulatório de clínica cirúrgica (situado, portanto, fora do centro cirúrgico).

Definiu-se como suspensa toda cirurgia programada que, por qualquer razão, não aconteceu na data agendada.

Uma graduanda de enfermagem realizou semanalmente a coleta de dados, utilizando instrumento elaborado 
especialmente para este fim, o qual contém os registros das cirurgias realizadas e suspensas.

Os dados relativos às cirurgias suspensas estão organizados em grupos de causas do cancelamento, tais como:

- condições pessoais: não comparecimento ou atraso do paciente e desistência;

- condições clínicas: alterações nos exames, mudança ou falta de condições clínicas, melhora do quadro clínico, infecções respiratórias, outras infecções, problemas cardiovasculares, pressão arterial sistêmica elevada;

- condições institucionais: não comparecimento do cirurgião, falta de anestesista, problemas com recursos materiais, falta de hemocomponentes, falta de vaga em UTI, falta de exames, falta de vaga na enfermaria, erros de agendamento, alteração na data, dificuldade de internação, falha na comunicação da equipe, falha no preparo adequado do paciente;

- outras causas e causas não mencionadas.

Estão ainda categorizados por especialidades cirúrgicas: pediátrica, geral, aparelho digestivo, oncológica, plástica, mamária, coloproctológica, otorrinolaringológica, ginecológica, urológica, cabeça e pescoço e outras.

Para a análise dos dados, realizou-se estatística descritiva de frequência simples. A taxa de suspensão de cirurgia foi calculada pelo número de cirurgias suspensas dividindo-se pelo total de cirurgias programadas em determinado período e multiplicado por cem.

Foram seguidas as diretrizes da Resolução do Conselho Nacional de Saúde no de 466/2012 do Conselho Nacional de Saúde, aprovado pelo Comitê de Ética do Hospital Universitário da Universidade Federal de Sergipe, sob CAAE $\mathrm{n}^{\mathrm{o}}$ 24871014.1.0000.5546, via plataforma Brasil.

\section{RESULTADOS}

No período estudado, das 1.600 cirurgias programadas, 1.287 foram realizadas e 313 suspensas, resultando, portanto, em uma taxa de cancelamento cirúrgico de 19,50\%.

As especialidades cirúrgicas que apresentaram as maiores frequências de suspensões cirúrgicas foram as cirurgias pediátricas $(26,8 \%)$, seguidas pelas oncológicas $(14,4 \%)$ e gerais $(13,4 \%)$. Apresentaram as menores taxas as especialidades cabeça e pescoço $(1,9 \%)$ e urológicas $(1,0 \%)$ (Tabela 1$)$.
Analisando-se as causas de suspensões de cirurgias, constatou-se que $50,8 \%$ das ocorrências estavam relacionadas às condições institucionais, e $43,5 \%$, ao paciente, seja por condições pessoais $(22,4 \%)$ ou condições clínicas (20,8\%) (Tabela 2).

Dentre as causas categorizadas como condições pessoais, destaca-se como a mais frequente o não comparecimento ou atraso do paciente. Das causas referentes às condições clínicas do paciente, identificou-se principalmente infecções do aparelho respiratório, seguida pela elevação da pressão arterial sistêmica e pela mudança ou falta de condições clínicas.

Entre as causas relacionadas às condições institucionais, foram mais elevadas e percentualmente semelhantes: problemas com recursos materiais, falta de vagas na enfermaria e não comparecimento do cirurgião. As menos frequentes foram: falta de anestesista, falta de vaga em UTI, falta de realização de exames e suspensão da cirurgia pelo anestesista.

\section{DISCUSSÃO}

Nesse estudo, a taxa de cancelamento de cirurgia utilizada no gerenciamento hospitalar (dado que traduz a eficiência das salas operatórias e que é considerado um indicador de qualidade dos serviços) foi semelhante às encontradas em pesquisas nacionais realizadas em hospitais de ensino nos

Tabela 1. Distribuição das frequências das cirurgias suspensas segundo as especialidades cirúrgicas, em Aracaju, Sergipe, 2013.

\begin{tabular}{|l|c|c|}
\hline Especialidade cirúrgica & $\mathbf{n}$ & $\%$ \\
\hline Pediátrica & 84 & 26,8 \\
\hline Oncológica & 45 & 14,4 \\
\hline Geral & 42 & 13,4 \\
\hline Aparelho digestivo & 31 & 9,9 \\
\hline Plástica & 28 & 8,9 \\
\hline Mamária & 22 & 7,0 \\
\hline Coloproctológica & 18 & 5,8 \\
\hline Ginecológica & 12 & 3,8 \\
\hline Otorrinolaringológica & 11 & 3,5 \\
\hline Cirurgia não mencionada & 11 & 3,5 \\
\hline Cabeça e pescoço & 6 & 1,9 \\
\hline Urológica & 3 & 1,0 \\
\hline Total & 313 & 100,0 \\
\hline
\end{tabular}


estados de São Paulo, Minas Gerais e Paraná, que identificaram taxas de 17,$3 ; 17,0$ e $14,1 \%$; respectivamente ${ }^{11-13}$.

Entretanto, quando comparadas com pesquisas internacionais, observa-se que as taxas estão muito acima das

Tabela 2. Distribuição das causas de suspensões cirúrgicas, segundo as condições pessoais, clínicas e institucionais, em Aracaju, Sergipe, 2013.

\begin{tabular}{|c|c|c|}
\hline Causas de suspensão de cirurgias & $\mathbf{n}$ & $\%$ \\
\hline \multicolumn{3}{|l|}{ Condições pessoais } \\
\hline Não comparecimento ou atraso do paciente & 70 & 22,4 \\
\hline Desistência & 1 & 0,3 \\
\hline Subtotal & 71 & 22,7 \\
\hline \multicolumn{3}{|l|}{ Condições clínicas } \\
\hline Infecções do aparelho respiratório & 24 & 7,7 \\
\hline Pressão arterial sistêmica elevada & 17 & 5,4 \\
\hline Mudança ou falta de condições clínicas & 10 & 3,2 \\
\hline Outras infecções & 6 & 1,9 \\
\hline Melhora do quadro clínico & 4 & 1,3 \\
\hline Alterações nos exames & 3 & 1,0 \\
\hline Problemas cardiovasculares & 1 & 0,3 \\
\hline Subtotal & 65 & 20,8 \\
\hline \multicolumn{3}{|l|}{ Condições institucionais } \\
\hline Problemas com recursos materiais & 27 & 8,6 \\
\hline Falta de vagas na enfermaria & 21 & 6,8 \\
\hline Não comparecimento do cirurgião & 20 & 6,3 \\
\hline Erros de agendamento & 18 & 5,8 \\
\hline Suspensão da cirurgia pelo cirurgião & 17 & 5,4 \\
\hline Falha no preparo adequado do paciente & 14 & 4,5 \\
\hline Dificuldade de internação & 11 & 3,5 \\
\hline Alteração de data & 10 & 3,2 \\
\hline Falta de hemocomponentes & 7 & 2,2 \\
\hline Falta de anestesista & 4 & 1,3 \\
\hline Falta de vaga em UTI & 4 & 1,3 \\
\hline Falta de exames & 3 & 1,0 \\
\hline Suspensão da cirurgia pelo anestesiologista & 2 & 0,6 \\
\hline Falha na comunicação da equipe & 1 & 0,3 \\
\hline Subtotal & 159 & 50,8 \\
\hline Outras causas & 11 & 3,5 \\
\hline Causas não mencionadas & 7 & 2,2 \\
\hline Total & 313 & 100,0 \\
\hline
\end{tabular}

encontradas em hospitais universitários do exterior. Pode-se citar: 8,80\% na Coreia; 4,40\% no Líbano; 0,37\% em Taiwan e $0,21 \%$ na China ${ }^{8,14-16}$. Tal achado é lamentável, visto que se traduz, em parte, por baixa qualidade dos serviços de saúde prestados. Isso porque os cancelamentos têm como consequência imediata, entre outros fatores, a não otimização do uso das salas operatórias.

Quanto às especialidades cirúrgicas, os procedimentos pediátricos apresentaram a maior taxa de suspensão, mais de quatro vezes acima de estudos realizados no sudeste do país; estudos paulistas, por exemplo, apresentaram taxas de 14,4 e $6,4 \%{ }^{13,11}$. Pesquisadores atribuem as suspensões de cirurgias pediátricas à comunicação ineficaz entre profissionais e familiares das crianças. Afirmam, ainda, que as informações são superficiais e incompletas, deixando dúvidas e gerando sentimentos como ansiedade, medo, insegurança e angústia ${ }^{11}$.

Dentre as causas de suspensão de cirurgia, o maior percentual encontrado foi o de não comparecimento ou atraso do cliente. Esse resultado é semelhante ao apontado por pesquisas realizadas em instituições de ensino de cidades do sudeste do Brasil, as quais revelam percentuais de 18,1 e $18,5 \%^{17,11}$.

Chama atenção o percentual encontrado em Aracaju $(22,4 \%)$, cidade do nordeste brasileiro, expressivamente menor do que a taxa encontrada em estudo realizado em hospital de Fortaleza $(39,9 \%)^{5}$. Tal dado pode ser justificado pelo fato de que, na instituição em estudo, as operações são agendadas poucos dias antes da realização do procedimento, e na outra instituição o agendamento é feito com bastante antecedência.

No presente estudo, o percentual observado na variável não comparecimento ou atraso do cliente é mais elevado do que o de estudos realizados no Reino Unido (6,8\%), Líbano $(11,1 \%)$ e Índia $(4,1 \%)^{18,14,19}$. O não comparecimento do paciente gera desperdício de material, tempo e pessoal, além de perda de oportunidade de agendamento para outro paciente; desse modo, o serviço do bloco cirúrgico e das unidades afins não é otimizado ${ }^{20}$.

Assim, é necessária uma melhor investigação acerca da razão de não comparecimento do cliente para o planejamento de estratégias de intervenção. Estudo sobre absenteísmo do usuário demonstra a importância de realização da busca ativa para confirmar a presença do usuário na cirurgia e/ou de modificações no sistema de agendamento dos procedimentos cirúrgicos, pois algumas são agendadas com bastante antecedência ${ }^{11,17}$. 
No presente estudo, as condições clínicas foram a terceira maior causa de cancelamentos, taxa aproximadamente 2 vezes maior do que a encontrada em hospital universitário paulista, e 1,6 vezes menor do que a encontrada em hospital de grande porte em Taiwan ${ }^{11,8}$.

Segundo a literatura, é evidente que muitos dos casos cancelados poderiam ter sido reconhecidos anteriormente, possibilitando tomadas de medidas corretivas. É consenso entre os autores que a existência de ambulatórios de anestesia e visitas pré-operatórias reduzem o número de suspensões cirúrgicas, por permitirem a previsão de possíveis complicações clínicas ${ }^{8,20}$.

As condições institucionais contribuem de forma significativa para suspensão de procedimentos cirúrgicos na unidade de saúde estudada, sendo a causa responsável pela metade dessas e aparecendo como o segundo grupo mais frequente. Isso pode ser explicado por problemas de cunho organizacional, erros no agendamento, impossibilidade de comparecimento do cirurgião e alteração de data. Resultado igual foi obtido por estudo realizado em hospital escola do Paraná, o qual constatou que a falta de materiais e equipamentos específicos foi também a segunda maior causa de cancelamento de cirurgia, o que leva a crer que esse pode ser um desafio às instituições públicas ${ }^{12}$. Nestas, o processo de compra é lento e burocrático e o sucateamento dos recursos existentes é cada vez maior ${ }^{12}$.

A frequência das causas de suspensão de procedimento cirúrgico é diferente e depende da realidade de cada instituição, porém, os problemas encontrados são comuns e devem ser monitorados através de indicadores que vão subsidiar o planejamento e a avaliação de ações de melhoria.

Embora tenha sido constatado, no presente estudo, que o item falha de comunicação da equipe apresentou um percentual baixo, a comunicação ineficaz pode ser a causa central e subliminar de vários dos itens revelados, e o impacto pode ser mais relevante do que os números apresentados.

\section{CONCLUSÃO}

O estudo encontrou uma taxa global de cancelamento de procedimentos cirúrgicos semelhante às nacionais e superior às internacionais. As maiores frequências de suspensão ocorreram nas cirurgias pediátricas, oncológicas e gerais. Foram reveladas 23 causas para o cancelamento de cirurgias na instituição investigada, dentre elas destacaram-se o absenteísmo do paciente e as condições institucionais, representadas principalmente por problemas com recursos materiais, humanos e ligados à organização do serviço.

Assim, o monitoramento dos indicadores relacionados à suspensão de cirurgia deve ser contínuo e subsidiar o planejamento de estratégias de intervenção com vistas à redução da taxa de suspensão e consequente minimização dos transtornos causados para os clientes, familiares e instituição.

\section{REFERÊNCIAS}

1. Costa Junior AL, Doca FNP, Araújo I, Martins L, Mundim L, Penatti T, et al. Preparação psicológica de pacientes submetidos a procedimentos cirúrgicos. Estud Psicol. 2012;29(2):271-84.

2. Denton B, Viapiano J, Volgl A. Optimization of surgery sequencing and scheduling decisions under uncertainty. Health Care Manag Sci. 2007;10(1)13-24.

3. Morgan W, Bernardino E, Wolff LDG. Implications of cancellation of surgery in a surgery department: a descriptive-exploratory study. Online Braz J Nurs. 2010;9(1):13.

4. Organização Mundial de Saúde. Avaliação dos Programas de Saúde: normas fundamentais para sua aplicação no processo de gestação para o desenvolvimento nacional de saúde. Genebra: OMS; 1981.
5. Landim FM, Paiva FDS, Fiuza MLT, Oliveira EP, Pereira JG, Siqueira IA. Análise dos fatores relacionados à suspensão de operações em um serviço de cirurgia geral demédia complexidade. Rev Col Bras Cir. 2009;36(4):283-7.

6. Ebirim LN, Buowari DY, Ezike H.A. Causes of cancellation of elective surgical operations at a University Teaching Hospital. Afr J Med Med Sci. 2012;3(5):297-301.

7. Garcia ACKA, Fonseca FL. A problemática da suspensão cirúrgica: a perspectiva dos anestesiologistas. Rev Enferm UFPE on line. 2013;7(2):481-90.

8. Sung WC, Chou AH, Liao CC, Yang MW, Chang CJ. Operation Cancellation at Chang Gung Memorial Hospital. Chang Gung Med J. 2010;33(5):568-75. 
9. Aquino FMM, Vera LF, Pinto ACS. A suspensão de cirurgia e o processo de comunicação. Rev. pesqui. cuid. fundam. (Online). [Internet]. 2012;4(2):2998-3005. Disponível em: http://www.seer.unirio.br/ index.php/cuidadofundamental/article/view/1810/www.media.wix. com/ugd//e1973c_35f0d73449e2cbaddd742db5147dd1 c5.doc

10. Novaretti MCZ. Aplicação da análise causa raiz como ferramenta na gestão de segurança hospitalar. Rev Adm UFSM. 2014;7(3):442-5.

11. Macedo JM, Kano JA, Braga EM, Garcia MA, Caldeira SM. Cancelamento de cirurgias em um hospital universitário: causas e tempo de espera para novo procedimento. Rev SOBECC. 2013;18(1):26-34.

12. Nascimento LA, Tillvitz LR, Fonseca LF. Suspensão cirúrgica: o ângulo estatístico de um problema de repercussões humanas. Rev Enferm UFPE on line. 2013;7(esp):6592-600.

13. Barbosa MH, Miranda DMG, Andrade EV, Mattia AL. Análise da suspensão de cirurgias em um hospital de ensino. Enferm Glob. 2012;11(26):164-73.

14. Kaddoum R, Fadlallah R, Hitti E, El-Jardali F, El Eid G. Causes of cancellations on the day of surgery at a Tertiary Teaching Hospital. BMC Health Serv Res. 2016;16:259.
15. Kim KO, Lee J. Reasons for cancellation of elective surgery in a 500 -bed teaching hospital: a prospective study. Korean J Anesthesiol. 2014;67(1):66-7.

16. Chang JH, Chen KW, Chen KB, Poon KS, Liu SK. Case review analysis of operating room decisions to cancel surgery. BMC Surg. 2014;14:47.

17. Avila MAGD, Bocchi SCM. Confirmação de presença de usuário à cirurgia eletiva por telefone como estratégia para reduzir absenteísmo. Rev Esc Enferm USP. 2013;47(1):193-7.

18. Dimitriadis PA, lyer S, Evgeniou E. The challenge of cancellations on the day of surgery. Int J Surg. 2013;11(10):1126-30.

19. Talati S, Gupta A K, Kumar A, Malhotra S K, Jain A. An analysis of time utilization and cancellations of scheduled cases in the main operation theater complex of a tertiary care teaching institute of North India. J Postgrad Med. 2015;61(1):3-8.

20. Paschoal MLH, Gatto MAF. Taxa de suspensão de cirurgia em um hospital universitário e os motivos de absenteísmo do paciente à cirurgia programada. Rev Latino-Am Enferm. 2006;14(1):48-53. 